University of New Hampshire

University of New Hampshire Scholars' Repository

Physics Scholarship

Physics

$5-2014$

\title{
Chorus acceleration of radiation belt relativistic electrons during March 2013 geomagnetic storm
}

\author{
Fuliang Xiao \\ Changsha University of Science and Technology \\ Chang Yang \\ Changsha University of Science and Technology \\ Zhaoguo He \\ Chinese Academy of Sciences, Beijing \\ Zhenpeng Su \\ University of Science and Technology of China \\ Qinghua Zhou \\ Changsha University of Science and Technology
}

See next page for additional authors

Follow this and additional works at: https://scholars.unh.edu/physics_facpub

Part of the Physics Commons

\section{Recommended Citation}

Xiao, F., et al. (2014), Chorus acceleration of radiation belt relativistic electrons during March 2013 geomagnetic storm, J. Geophys. Res. Space Physics, 119, 3325-3332, doi:10.1002/2014JA019822.

This Article is brought to you for free and open access by the Physics at University of New Hampshire Scholars' Repository. It has been accepted for inclusion in Physics Scholarship by an authorized administrator of University of New Hampshire Scholars' Repository. For more information, please contact Scholarly.Communication@unh.edu. 


\section{Authors}

Fuliang Xiao, Chang Yang, Zhaoguo He, Zhenpeng Su, Qinghua Zhou, Yihua He, C A. Kletzing, W. S. Kurth, G. B. Hospodarsky, Harlan E. Spence, Geoffrey Reeves, H. O. Funsten, J. B. Blake, D. N. Baker, and J. R. Wygant 


\section{Journal of Geophysical Research: Space Physics}

\author{
BRIEF REPORT \\ 10.1002/2014JA019822 \\ Key Points: \\ - Initial RBSP correlated data of chorus \\ waves and relativistic electron fluxes \\ - A realistic simulation to examine \\ effect of chorus on relativistic \\ electron flux \\ - Chorus yields huge increases in \\ electron flux rapidly, consistent \\ with data
}

Correspondence to:

F. Xiao,

flxiao@126.com

\section{Citation:}

Xiao, F., et al. (2014), Chorus acceleration of radiation belt relativistic electrons during March 2013 geomagnetic storm, J. Geophys. Res. Space

Physics, 119, 3325-3332,

doi:10.1002/2014JA019822.

Received 25 JAN 2014 Accepted 28 APR 2014

Accepted article online 3 MAY 2014 Published online 23 MAY 2014

\section{Chorus acceleration of radiation belt relativistic electrons during March 2013 geomagnetic storm}

\author{
Fuliang Xiao ${ }^{1}$, Chang Yang ${ }^{1}$, Zhaoguo $\mathrm{He}^{2}$, Zhenpeng Su${ }^{3}$, Qinghua Zhou ${ }^{1}$, Yihua $\mathrm{He}^{1}$, \\ C. A. Kletzing ${ }^{4}$, W. S. Kurth ${ }^{4}$, G. B. Hospodarsky ${ }^{4}$, H. E. Spence ${ }^{5}$, G. D. Reeves ${ }^{6}$, H. O. Funsten ${ }^{7}$, \\ J. B. Blake ${ }^{8}$, D. N. Baker' ${ }^{\text {, and J. R. Wygant }}{ }^{10}$
}

${ }^{1}$ School of Physics and Electronic Sciences, Changsha University of Science and Technology, Changsha, China, ${ }^{2}$ Center for Space Science and Applied Research, Chinese Academy of Sciences, Beijing, China, ${ }^{3}$ CAS Key Laboratory of Geospace Environment, Department of Geophysics and Planetary Sciences, University of Science and Technology of China, Hefei, China, ${ }^{4}$ Department of Physics and Astronomy, University of lowa, lowa City, lowa, USA, ${ }^{5}$ Institute for the Study of Earth, Oceans, and Space, University of New Hampshire, Durham, New Hampshire, USA, ${ }^{6}$ Space Science and Applications Group, Los Alamos National Laboratory, Los Alamos, New Mexico, USA, ${ }^{7}$ ISR Division, Los Alamos National Laboratory, Los Alamos, New Mexico, USA, ${ }^{8}$ The Aerospace Corporation, Los Angeles, California, USA, ${ }^{9}$ Laboratory for Atmospheric and Space Physics, University of Colorado Boulder, Colorado, USA, ${ }^{10}$ School of Physics and Astronomy, University of Minnesota, Minneapolis, Minnesota, USA

Abstract The recent launching of Van Allen probes provides an unprecedent opportunity to investigate variations of the radiation belt relativistic electrons. During the 17-19 March 2013 storm, the Van Allen probes simultaneously detected strong chorus waves and substantial increases in fluxes of relativistic (2-4.5 MeV) electrons around $L=4.5$. Chorus waves occurred within the lower band $0.1-0.5 f_{c e}$ (the electron equatorial gyrofrequency), with a peak spectral density $\sim 10^{-4} \mathrm{nT}^{2} / \mathrm{Hz}$. Correspondingly, relativistic electron fluxes increased by a factor of $10^{2}-10^{3}$ during the recovery phase compared to the main phase levels. By means of a Gaussian fit to the observed chorus spectra, the drift and bounce-averaged diffusion coefficients are calculated and then used to solve a 2-D Fokker-Planck diffusion equation. Numerical simulations demonstrate that the lower-band chorus waves indeed produce such huge enhancements in relativistic electron fluxes within $15 \mathrm{~h}$, fitting well with the observation.

\section{Introduction}

The Earth's radiation belts dynamics are strongly associated with wave-particle interactions which determine local acceleration and loss of energetic particles [Thorne, 2010]. On 30 August 2012, two NASA Van Allen probes were launched into highly elliptical and low-inclination orbits [Mauk et al., 2012], in order for in-depth understanding of the processes which potentially yield dynamic evolution of the radiation belts. Different instruments on two Van Allen probes play different role in collecting data throughout the Van Allen probes orbit. The Relativistic Electron-Proton Telescope (REPT) instrument acts to measure the relativistic (1 MeV to $20 \mathrm{MeV}$ ) electrons [Baker et al., 2012]. The Electric and Magnetic Field Instrument Suite and Integrated Science (EMFISIS) is designed to collect data of wave electric and magnetic fields as well as DC magnetic fields [Kletzing et al., 2013; Wygant et al., 2013]. Currently, new advances have been made in our understanding of the radiation belt dynamics based on the latest observation of Van Allen probes. Baker et al. [2013] initially reported a new radiation belt relativistic electron ring which occurred between the locations 3-3.5 $R_{E}$ (the Earth's radius). Thorne et al. [2013a] proposed that the small pitch angle diffusion rates induced by hiss waves could account for the slow decay of relativistic electron ring. Shprits et al. [2013] found that there was no gyroresonance between relativistic electrons with low-latitudes waves, probably leading to such a long-lived electron ring. Reeves et al. [2013] made a detailed analysis of radial profiles of electron phase space density (PSD) and linked them to in situ accelerations in the heart of the radiation belts. Morley et al. [2013] used the PSD matching method to analyze error in electron PSD gathered by Van Allen probes in adiabatic invariant coordinates. Li et al. [2013] observed an extreme low-frequency (close to $20 \mathrm{~Hz}$ ) plasmaspheric hiss waves in the outer plasmasphere on 30 September 2012 when an strong electron injection event occurred. 

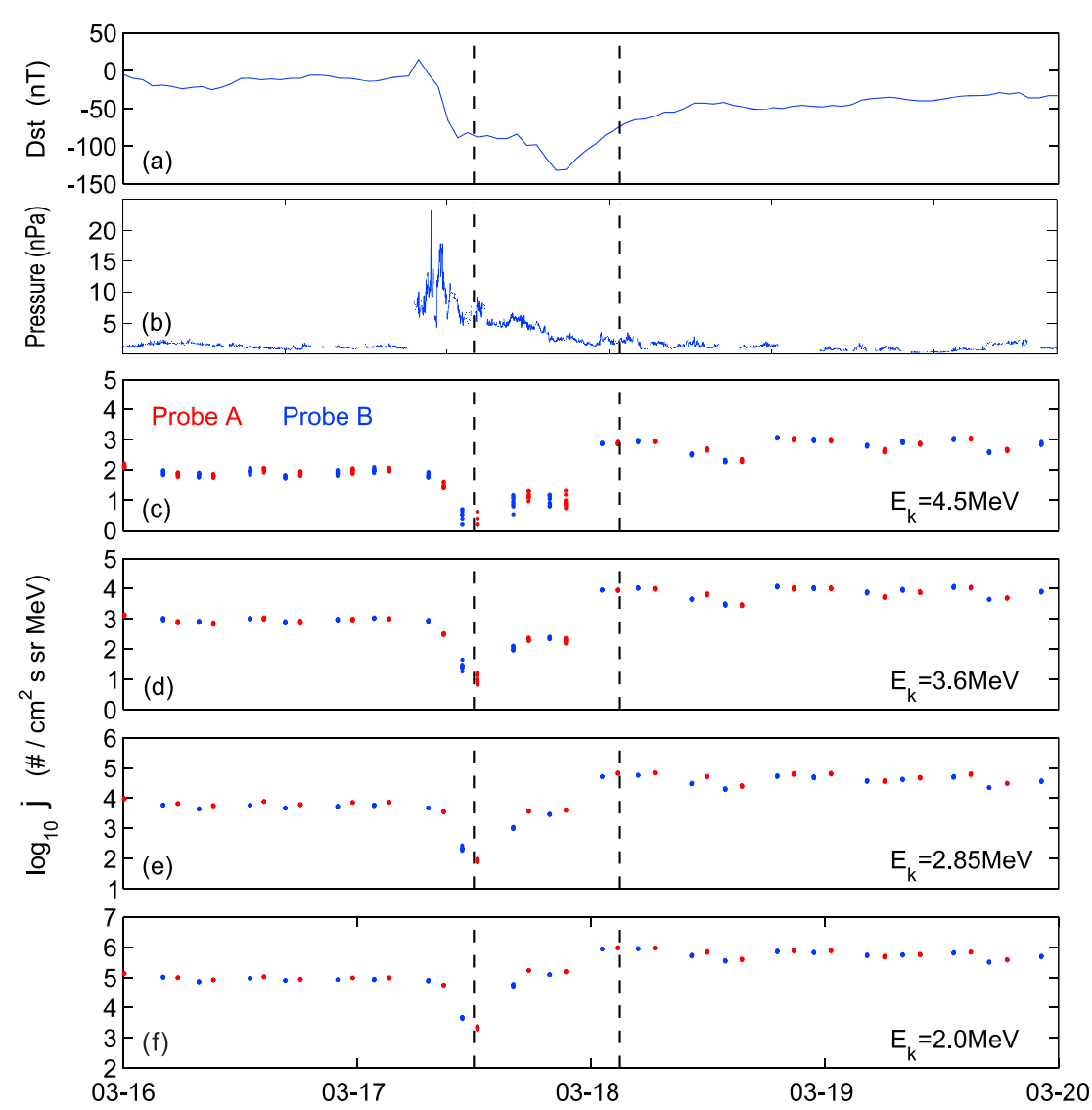

Figure 1. Van Allen probe data during 16-20 March 2013. (a) The Dst index. (b) Solar wind dynamic pressure. (c-f) Flux of electrons $(2-4.5 \mathrm{MeV}$ ) measured by ECT-REPT instrument at the location $L=4.5 \pm 0.02$. The pair of vertical dot lines indicate the simulating storm period: 12:00 UT on 17 March to 03:00 UT on 18 March.

On 17 March 2013, a strong magnetic storm occurred with minimum Dst -132 nT after a strong interplanetary shock encountered the Earth's magnetosphere. The REPT instrument observed substantial enhancements in fluxes of relativistic electrons above $2 \mathrm{MeV}$ at $L>3.5$. In the meanwhile, the EMFISIS instrument detected enhanced whistler mode chorus waves with frequencies from $\sim 100 \mathrm{~Hz}$ up to $\sim 10 \mathrm{kHz}$. Such simultaneous observations tend to suggest that chorus waves can be responsible for the flux enhancement of relativistic electrons, but this requires detailed data analysis and the corresponding numerical modeling. This is the main purpose of this study.

\section{Van Allen Probe Correlated Data}

Figure 1a shows the time history of geomagnetic activity Dst during 16-20 March 2013. The storm appears to consist of a "two-step" main phase where Dst at first dropped rapidly down to $-89 \mathrm{nT}$ at 10:30:00 UT and remained almost the same level for a few hours and then dropped rapid again down to $-132 \mathrm{nT}$ at 20:30:00 UT on 17 March. Figure $1 \mathrm{~b}$ shows the solar wind dynamic pressure. Figures 1c-1f show the dynamical evolution of outer radiation belt relativistic (2-4.5 MeV) electrons observed by Energetic particle, Composition, and Thermal plasma (ECT)-REPT instrument onboard both Van Allen probes when they stayed around the location $L=4.5$ on the nightside. Obviously, relativistic electron fluxes dropped rapidly by a factor of 1-2 orders at the onset of the storm: 06:00 UT on 17 March. Such rapid flux reductions should be attributed to either the adiabatic loss (Dst effect) or wave-particle interaction (e.g., hiss-electron interaction) or the magnetopause shadowing due to inward motion of the magnetopause during the geomagnetic storm. During the second-step main phase, Dst still decreased but electron fluxes started to increase and reached the level comparable to the previous storm level. This is perhaps more associated with the nonadiabatic process, e.g., wave-particle interaction. During the recovery phase from 20:30:00 UT on 17 March to 03:00 UT on 18 March, electron fluxes continued to increase and reached the peak values by about 3 (for 


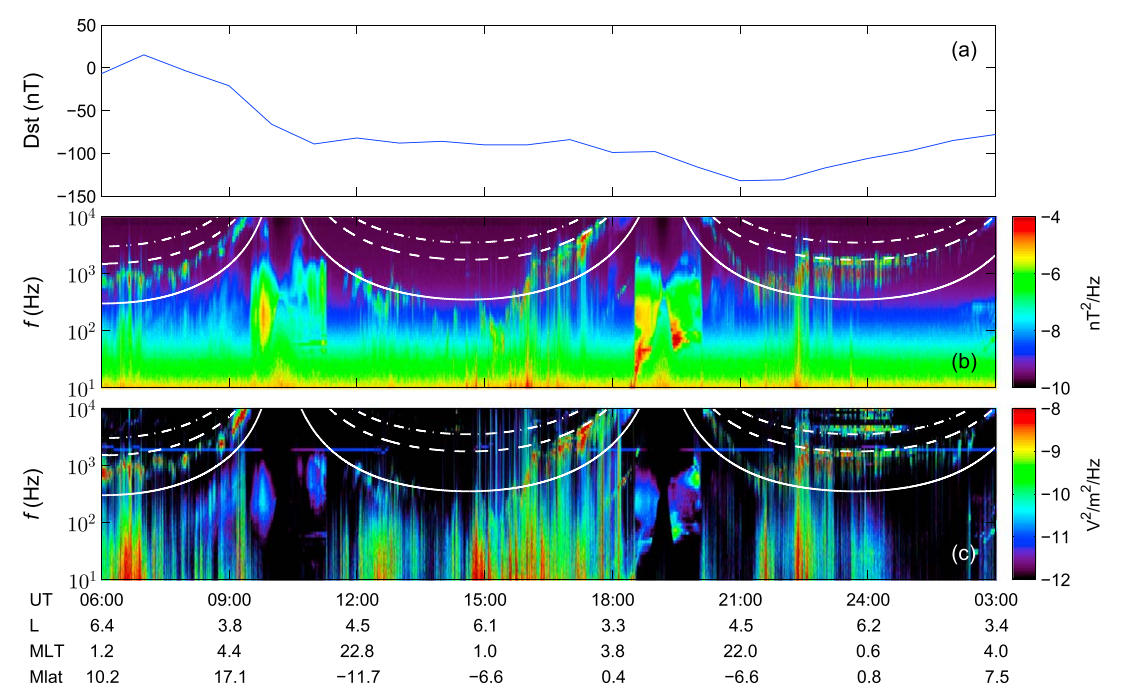

Figure 2. (a) The Dst index. (b) magnetic field spectral density and (c) electric field spectral density measured by EMFISIS instrument onboard Van Allen probe A during the period of 06:00 UT on 17 March to 03:00 UT on 18 March. The white lines obtained by the ECT-MagEIS magnetic field data represent $0.1 f_{c e}$ (solid), $0.5 f_{c e}$ (dashed), and $f_{c e}$ (dash-dotted).

2-3.6 MeV) or 2 (for 4.5 MeV) orders of magnitude higher than the main phase levels. The flux enhancements should come from both nonadiabatic process and Dst effect. However, Dst effect appears to play a less important role since $\Delta D s t \approx 55 \mathrm{nT}$ during the period from 12:00 UT on 17 March to 03:00 UT on 18 March. Moreover, using the relation for adjusting electron distribution due to Dst effect [Li et al., 2009], we made a rough check and found that the fluxes basically increase by a few times in considering Dst effect. At the same time, the EMFISIS instrument observed intensified whistler wave activities from 06:00 UT on 17 March to 03:00 UT on 18 March on the nightside (Figure 2). In particular, during the periods: 06:00-09:00 UT, 15:00-18:00 UT, and 21:00-02:00 UT, the distinct wave frequency was scaled with gyrofrequency of electron $f_{c e}$ and stayed between 0.1 and $0.5 f_{c e}$, characteristic of the lower band of chorus wave. Nevertheless, there is no direct observation of whistler waves on the dayside around the location $L=4.5$ because two Van Allen probes traveled near the perigee $L<2$. We also check the data from Time History of Events and Macroscale Interactions during Substorms spacecraft and find no obvious whistler mode wave activity around the location $L=4.5$ on the dayside. Since chorus waves are proposed to be a leading mechanism responsible for stochastic acceleration of energetic electrons [Summers et al., 1998; Horne et al., 2005a, 2005b; Thorne et al., 2013b], it is expected that the observed chorus waves should account for the temporal evolution of relativistic electrons in this event, which will be presented in detail in the following.

\section{Numerical Modeling}

Here we focus on the storm time period from 12:00 UT on 17 March to 03:00 UT on 18 March, when strong chorus waves and significant enhancements in fluxes of relativistic (2-4.5 MeV) electrons were simultaneously observed roughly in 2100 to 0300 magnetic local time (MLT). During the gyroresonance between chorus waves and electrons with the momentum $p$ (or the velocity $v$ ) and the equatorial pitch angle $\alpha_{e}$ the evolution of phase space density (PSD) $f$ can be described by a 2-D bounce-averaged Fokker-Planck equation as follows [Kozyra et al., 1994; Albert, 2004]:

$$
\begin{aligned}
\frac{\partial f}{\partial t}= & \frac{1}{G p} \frac{\partial}{\partial \alpha_{e}}\left[G\left(\left\langle D_{\alpha \alpha}\right\rangle \frac{1}{p} \frac{\partial f}{\partial \alpha_{e}}+\left\langle D_{\alpha p}\right\rangle \frac{\partial f}{\partial p}\right)\right] \\
& +\frac{1}{G} \frac{\partial}{\partial p}\left[G\left(\left\langle D_{p \alpha}\right\rangle \frac{1}{p} \frac{\partial f}{\partial \alpha_{e}}+\left\langle D_{p p}\right\rangle \frac{\partial f}{\partial p}\right)\right]
\end{aligned}
$$

here $T\left(\alpha_{e}\right)=v \tau_{b}\left(\alpha_{e}\right) /\left(4 L R_{E}\right) \approx 1.30-0.56 \sin \alpha_{e}$ gives the normalized bounce time with $\tau_{b}$ being the bounce time in a dipole magnetic field, $G=p^{2} T\left(\alpha_{e}\right) \sin \alpha_{e} \cos \alpha_{e},\left\langle D_{\alpha \alpha}\right\rangle_{,}\left\langle D_{p p}\right\rangle$, and $\left\langle D_{\alpha p}\right\rangle=\left\langle D_{p \alpha}\right\rangle$ stand for bounce-averaged pitch angle, momentum, and cross diffusion coefficients, respectively.

Before proceeding the calculation of the chorus-induced bounce-averaged diffusion coefficients, we need to specify the chorus wave power distributed in wave frequency and wave normal angle. A typical 


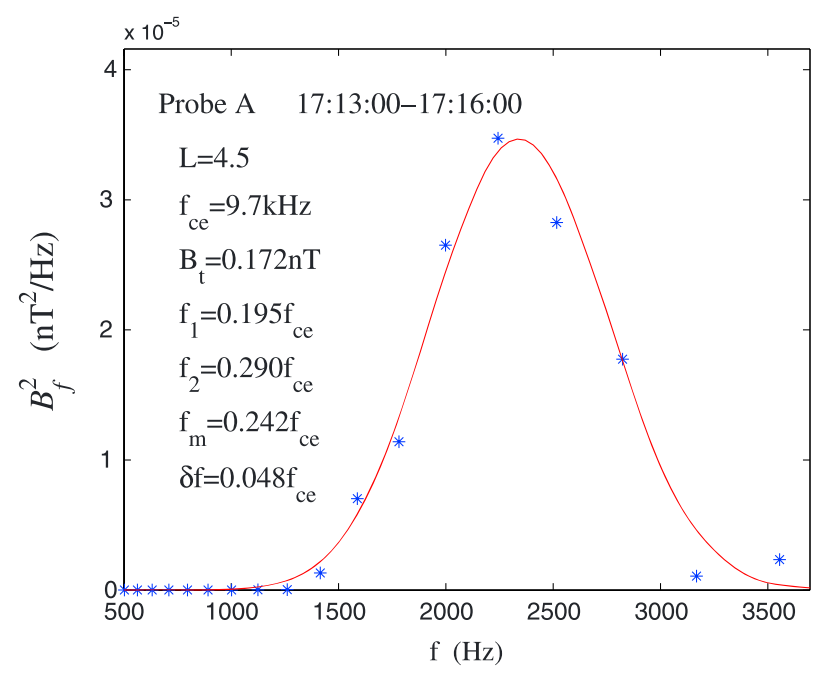

Figure 3. The modeled Gaussian fit (solid) to the observed wave spectra (dot) over a 3 min period 17:13:00-17:16:00 UT on 17 March, together with the corresponding fitting wave parameters.
Gaussian distribution is usually used to model the wave spectral density as a function of frequency and normal angle [Lyons et al., 1972]. Figure 3 shows a least squares Gaussian fit (solid) to the observed chorus spectral intensity (dot) by Van Allen probe A over a 3 min period 17:13:00-17:16:00 UT on 17 March, together with the corresponding best fitting values of parameters: the center frequency $f_{m}=0.242 f_{c e}$, the half width $\delta f=0.048 f_{c e}$, the lower frequency $f_{1}=0.195 f_{c e}$, and the upper frequency $f_{2}=0.29 f_{c e}$. Since the peak growth rate of whistler mode chorus waves tends to occur at the field-aligned direction [Horne et al., 2003], values of the wave normal angle $(X=\tan \theta)$ are chosen as follows[Glauert and Horne, 2005]: the lower angle $X_{1}=0$, the upper angle $X_{2}=1$, the half width $X_{\omega}=0.577 \mathrm{~m}$ and the peak $X_{m}=0$. Based on the measurements of the upper hybrid frequency, the nightside equatorial ratio of the electron plasma frequency to the gyrofrequency $f_{p e} / f_{c e}$ is taken 3.8 , comparable to the density model [Sheeley et al., 2001]. We assume that the electron number density and the wave spectral intensity remain constant along the dipolar geomagnetic field line. We consider contribution from harmonic resonances up to $n= \pm 5$ [Xiao et al., 2009] and the maximum occurrence latitude of chorus waves $\lambda_{m}=15^{\circ}$ (Figure 2).

Figure 4 displays three diffusion coefficients induced by the nightside chorus waves as a function of energy and pitch angle. Obviously, diffusion coefficients roughly increase as the equatorial pitch angle increases (except approaching $90^{\circ}$ ) but decrease as the kinetic energy increases. This probably explains the fact that the enhancement in electron flux occurs primarily at higher pitch angles, and such flux enhancement becomes smaller and slower at higher energies (Figure 1). The cross diffusion term (contributing to the pitch angle and energy diffusion) is higher than (or comparable to) the momentum diffusion term (controlling
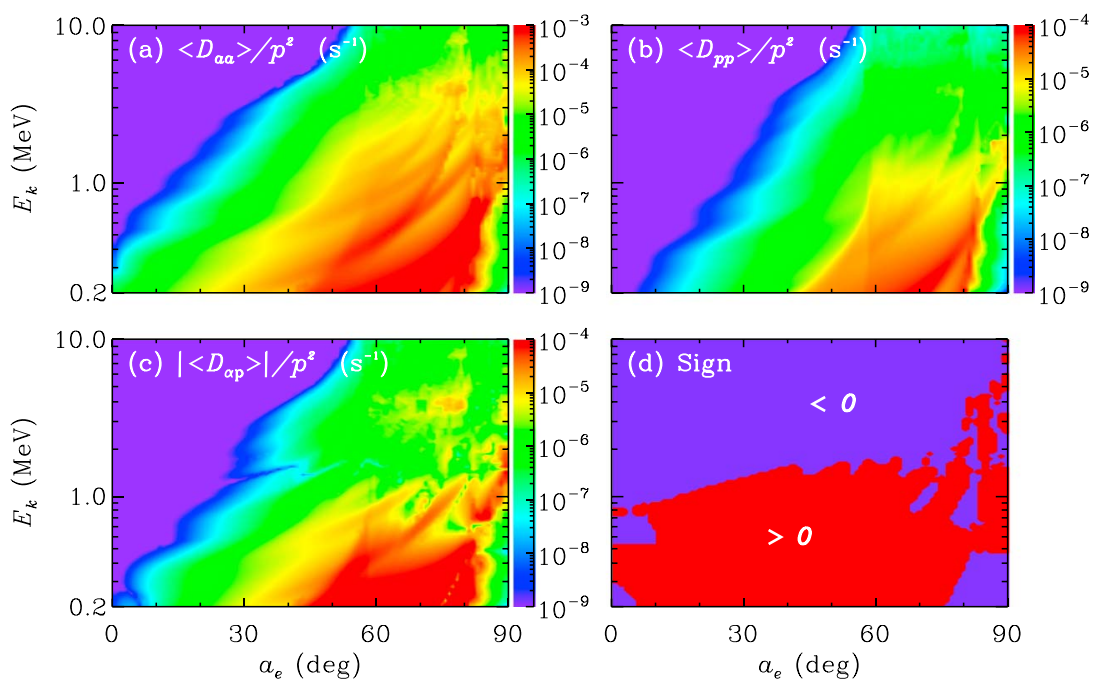

Figure 4. Bounce-averaged diffusion coefficients of (a) pitch angle, (b) momentum, and (c) cross in units of $s^{-1}$, as a function of energy and pitch angle. (d) The sign of the cross diffusion coefficient. 


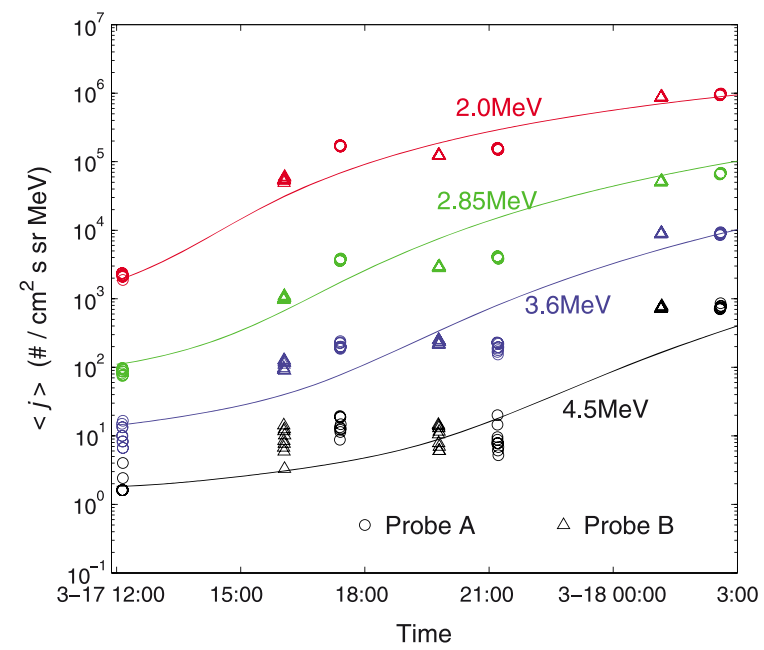

Figure 5. Comparison between the observed (discrete) and modeled (solid) pitch angle averaged differential fluxes variation with time from 12:00 UT on 17 March to 03:00 UT on 18 March. Note: there are more than one point and a spread at a time due to the data collected by probes $\mathrm{A}$ and $\mathrm{B}$ in the region $L=4.5 \pm 0.02$. the energy diffusion), changing the sign rapidly and alternatively particularly in the region above $\sim 60^{\circ}$ and $\sim 1 \mathrm{MeV}$. Furthermore, incorporation of all three diffusion rates allows efficient acceleration of relativistic (2-4.5 MeV) to occur at higher pitch angles, yielding the corresponding enhancements in relativistic electron fluxes on a time scale from a few hours to tens of hours.

Using standard finite difference methods to solve the Fokker-Planck diffusion equation (1) often encounters the unstable numerical problems if the rapidly varying cross diffusion terms are incorporated [Albert, 2004]. A variable transformation technique [Albert and Young, 2005] and a Monte Carlo method [Tao et al., 2008] were proposed to overcome this unstable problem. Here we adopt the hybrid finite difference method

[Xiao et al., 2009], in which the diagonal and off-diagonal (cross) diffusion coefficients are treated by an implicit scheme and an alternative direction implicit scheme, respectively. The numerical grid is chosen as $91 \times 91$ and uniform in pitch angle and natural logarithm of momentum, allowing no negative results occurring.

Solution of the diffusion equation (1) also needs to choose the appropriate and realistic initial and boundary conditions in order for a realistic simulation of this event. Boundary conditions in pitch angle are taken $f=0$ at $\alpha_{e}=0$ and $\partial f / \partial \alpha_{e}=0$ at $\alpha_{e}=90^{\circ}$. For the energy boundary conditions, $f$ is assumed to remain constant at the lower boundary $0.2 \mathrm{MeV}$ and the upper boundary $10 \mathrm{MeV}$, respectively.

Considering that energetic particles in tenuous and collisionless space plasmas often display a nonMaxwellian power law tail distribution [Vasyliunas, 1968; Viñas et al., 2005], we model the initial condition by a kappa-type distribution function of energetic electrons as follows [Xiao, 2006; Xiao et al., 2008]:

$$
f_{0}^{\kappa}\left(p, \sin \alpha_{e}\right)=\frac{n_{e} \Gamma(\kappa+l+1)}{\pi^{3 / 2} \theta^{3} \kappa^{(I+3 / 2)} \Gamma(I+1) \Gamma(\kappa-1 / 2)}\left(\frac{p \sin \alpha_{e}}{\theta}\right)^{2 l}\left[1+\frac{p^{2}}{\kappa \theta^{2}}\right]^{-(\kappa+l+1)}
$$

here $n_{e^{\prime}} l, \kappa$, and $\Gamma$, respectively, represent the number density, the loss-cone index, the spectral index, and the gamma function. The effective thermal parameter $\theta^{2}$ is scaled by the electron rest mass energy $m_{e} c^{2}$ $(\sim 0.512 \mathrm{MeV})$.

Using the fitting procedure in the previous work [Xiao et al., 2008], those parameters in (2) can be straightforward specified by comparing the data from the REPT instrument with the calculated differential flux $j$ by the conversion $j=p^{2} f$. We find the best fitting values of parameters: $\theta^{2}=0.4(\sim 200 \mathrm{keV}), I=0.5, \kappa=5$, and $n_{e}=0.0049 \mathrm{~cm}^{-3}$.

Since the nightside chorus emission occupies roughly $25 \%$ of the whole MLT region, energetic electrons only encounter the nightside chorus emission for about $25 \%$ in their drifting orbit around the Earth. Hence, we apply $25 \%$ drift averaging on those diffusion rates in Figure 4 as input to calculate PSD evolution due to the nightside chorus waves and then simulate the evolution of the differential flux. Moreover, considering that electron flux or PSD is a function of both energy and pitch angle, for convenience, we average the flux over the pitch angle $\alpha_{e}$ with the relation $\langle j\rangle=\frac{2}{\pi} \int_{0}^{\pi / 2} j \sin \alpha_{e} d \alpha_{e}$ to compare with the observation. In Figure 5, we plot the corresponding realistic simulation of the flux evolution at four indicated energies $E_{k}=2.0 \mathrm{MeV}, 2.85 \mathrm{MeV}$, 3.6 MeV, and 4.5 MeV with the observation (Figure 1). The discrete symbols represent the observational data collected by probes $A$ and $B$, and the solid line denotes the simulated 

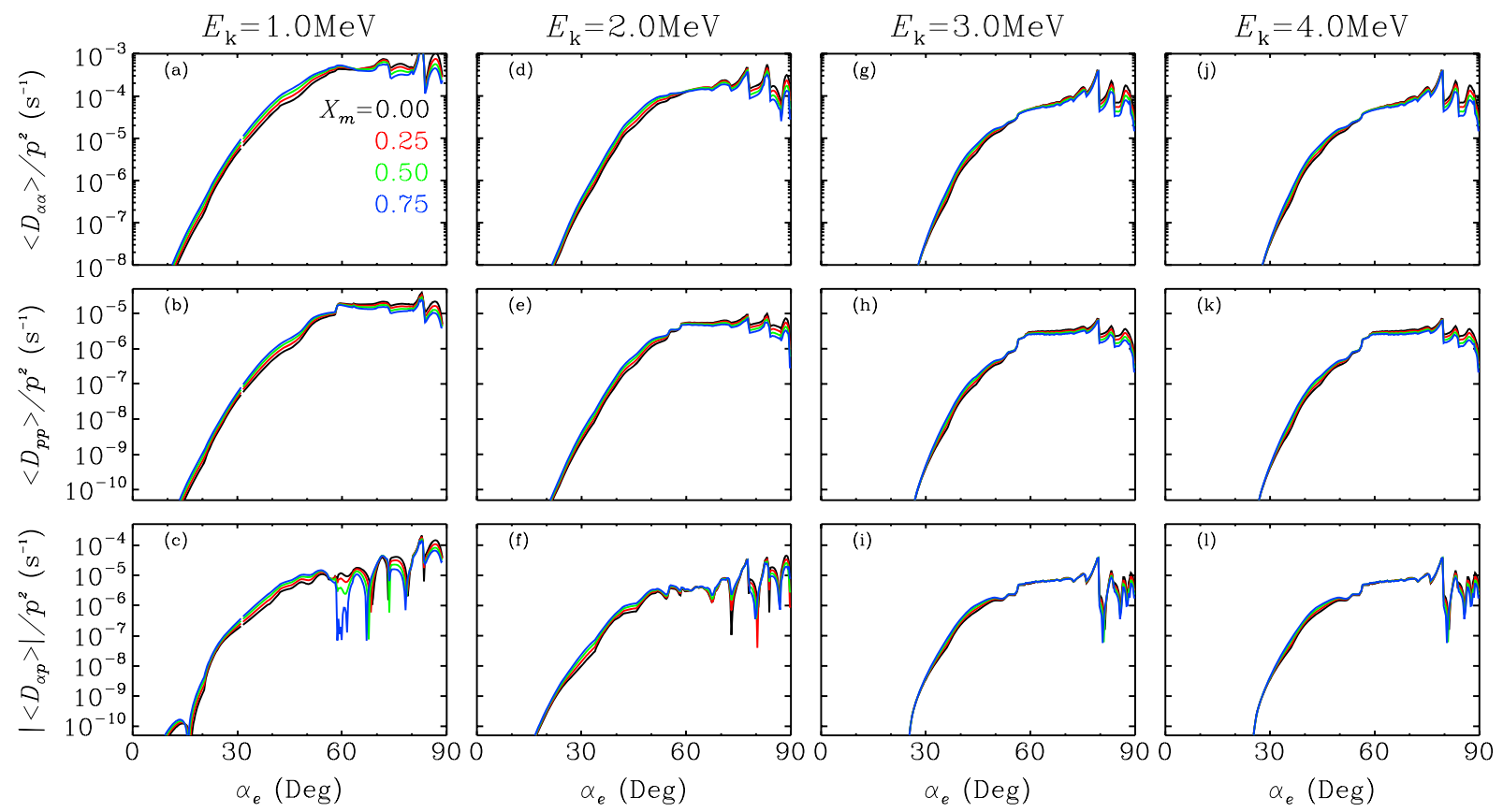

Figure 6. Diffusion coefficients for different indicated peak angles $X_{m}=0$ (black), 0.25 (red), 0.5 (green), and 0.75 (blue). The other wave parameters are the same as those in Figure 4.

differential flux $\langle j\rangle$. The modeling starts at 12:00 UT on 17 March and stops at 03:00 UT on 18 March, respectively, corresponding to the lowest flux level in the main phase and the highest flux level in the recovery phase. Obviously, the simulation shows a remarkably good agreement with the data that the averaged flux of relativistic increases substantially by a factor of $\sim 10^{3}$ at energies between 2-3.6 MeV and $\sim 10^{2}$ at energy $4.5 \mathrm{MeV}$ within about $15 \mathrm{~h}$. The electron loss in the simulation period probably comes from the drift loss to the magnetopause boundary. As shown in Figure $1 \mathrm{~b}$, a relatively weak solar wind pressure occurs in the simulation period, allowing the magnetopause boundary to stay outside in the region 8.5-11 $R_{E}$ [ Lin et al., 2010], thus minimizing the electron loss to boundary [Kataoka and Miyoshi, 2008]. Consequently, such solar wind condition is favorable for the dramatic chorus-driven acceleration. This is analogous to the previous study [Thorne et al., 2013b] that they similarly analyzed electron acceleration during the 9 October 2012 magnetic storm.

It should be mentioned that a least squares Gaussian fit to the observed chorus spectral intensity is performed over a specific 3 min period 17:13:00-13:16:00 UT on 17 March, corresponding to the maximum amplitude and perhaps the broadest band of the observed chorus around the location $L=4.5$. We then use those wave parameters throughout the modeled time period due to the lack of synoptic coverage by the Van Allen probes. If different 3 min intervals are used, the wave parameters particularly the wave amplitude and wave frequency change. In general, PSD evolution of electrons is essentially controlled by the diffusion coefficients associated with the wave magnetic amplitude and the wave frequency range. Because diffusion coefficients are positively proportional to the square of wave magnetic amplitude, and the number of resonant electrons increases (or decreases) with the frequency range increases (or decreases), the PSD evolution increases (or decreases) when the wave magnetic amplitude and the frequency range increases (or decreases). Hence, if chorus stayed at the observed intensity for less than $100 \%$ of the modeled time interval, the wave amplitude should decrease and then the timescale for acceleration should increase. However, this will not change the basic properties of wave-particle interaction.

Moreover, in Figure 6, we provide some calculations by changing the peak wave normal angle $X_{m}$ to investigate the sensitivity of PSD variation to $X_{m}$. It is shown that all the diffusion coefficients are very close for different wave peak angles. Xiao et al. [2010] investigated the electron PSD variation versus wave normal angles and demonstrated that a parallel $(X=0)$ chorus propagation approximation could provide reasonable results within mild and medium wave normal angles. Recently, Ding et al. [2013] made a detailed 
calculation how chorus-electron interaction was sensitive to the peak wave angles. They found that differences in diffusion coefficients were negligible within mild and medium wave normal angles but obvious for very oblique wave angles. This is reasonable since the diffusion coefficients are primarily determined by $X_{m}$ instead of the peak angle $\theta_{m}$. Within mild and medium wave normal angles, $X_{m}$ does not change much even $\theta_{m}$ changes from 0 to tens of degrees. Hence, in cases of interest, the results will not change much for different peak normal angles.

\section{Summary}

We have provided simultaneous observation of enhanced whistler mode waves and flux evolution of relativistic electrons around $L=4.5$ observed by two Van Allen probes on the nightside during the 17-19 March 2003 storm. Chorus waves stayed within the lower band $0.1-0.5 f_{c e}$, with a peak spectral density $\sim 10^{-4} \mathrm{nT}^{2} / \mathrm{Hz}$. In the meanwhile, substantial enhancements in fluxes of relativistic (2-4.5 MeV) electrons are found to occur by a factor of $10^{2}$ to $10^{3}$ during the period 12:00 UT on 17 March to 03:00 UT on 18 March, corresponding to intensified chorus waves.

In order to reveal the inherent relation between chorus and increases in fluxes, we have calculated bounce-averaged diffusion coefficients of momentum, pitch angle, and cross based on those realistic wave parameters obtained by a Gaussian least squares fit. When the equatorial pitch angle increases or the electron energy decreases, diffusion terms roughly increase, reasonably explaining the fact that the flux enhancement tends to take place at higher pitch angles but becomes smaller and slower at higher energies. Moreover, the cross diffusion term which contributes to pitch angle and energy diffusion is higher than (or comparable to) the momentum diffusion term which controls the energy diffusion, allowing the cross term also playing a key role in chorus-electron interaction.

Applying 25\% drift averaging on those diffusion coefficients to solve a 2-D bounce-averaged Fokker-Planck diffusion equation, we have presented a realistic simulation of the flux evolution of relativistic electrons at four indicated energies: $2 \mathrm{MeV}, 2.8 \mathrm{MeV}, 3.6 \mathrm{MeV}$, and 4.5 MeV. Modeled results fit well with the observed data that the pitch angle averaged flux of relativistic increases substantially by a factor of $10^{2}$ to $10^{3}$ within about $15 \mathrm{~h}$. This provides a direct observational evidence for chorus-driven acceleration of relativistic electrons in the radiation belts.

It should be mentioned that the current study focuses on whether chorus waves can produce efficient acceleration of relativistic electrons but is not intended to identify the relative contributions of in situ acceleration and radial diffusion. The inward radial diffusion can also yield the observed increase of relativistic electrons if a positive PSD radial gradient occurs during enhanced ultralow frequency wave activities [Li et al., 1997]. This was demonstrated by Tu et al. [2009] that the relative contributions to acceleration of relativistic electrons from radial diffusion and in situ acceleration varied from storm to storm. However, the radial diffusion coefficient decreases rapidly with decreasing L [Li et al., 2009; Brautigam and Albert, 2000], allowing smaller contribution of radial diffusion in the lower $L$ location [Xiao et al., 2010]. We provide some estimates (not shown for brevity) for the role of radial diffusion on electron acceleration using our previous 3-D diffusion code [Xiao et al., 2010]. We use the chorus wave parameters comparable to those in this study and the same radial diffusion coefficient $D_{L L}$ from a data-based $K_{p}$-dependent empirical relation [Brautigam and Albert, 2000]. We find that the difference between the results from chorus alone and chorus + radial diffusion are small since the radial diffusion is very weak at location $L=4.5$ due to $D_{L L} \approx L^{10}$. In particular, PSD evolutions for energy above $1.0 \mathrm{MeV}$ near pitch angle $90^{\circ}$ due to chorus alone are a few times higher than those due to chorus + radial diffusion after $15 \mathrm{~h}$. However, a realistic 3-D modeling including wave-particle interaction and radial diffusion requires the knowledge on a realistic global distribution of electromagnetic waves and the development of radial diffusion coefficients based on ultralow frequency wave power. Brautigam and Albert [2000] also demonstrated that the inward radial diffusion was efficient in acceleration of $<1 \mathrm{MeV}$ electrons but hard to account for the gradual increase of $>1 \mathrm{MeV}$ electrons during the recovery phase. In addition, as performed by Reeves et al. [2013], even with the high-energy resolution data available from Van Allen probes, determining the PSD radial profile still requires real-time data of magnetic field through different $L$ shells, detailed calculations of three adiabatic invariants: $\mu, J$, and $L^{*}$ in terms of $p$ and $\alpha$, and the conversion of the differential flux into PSD by $j=p^{2} f$. This is beyond the scope of this study but deserves a future work. 


\section{Acknowledgments}

The data for this paper are available at the following CDAWeb and RBSP websites: http://cdaweb.gsfc.nasa. gov/istp_public/,omni_hros_1min 20130316000000_20130320000000. cdf, and omni2_h0s_mrg1hr_ 20130316003000_20130320003000. cdf; ECT, http://www.rbsp-ect. lanl.gov/data_pub/,rbspa_rel02 ect-rept-sci-L2_20130316_v3.1.0. cdf, rbspa_rel02_ect-rept-sci-L2_ 20130317_v3.1.0.cdf,rbspa rel02 ect-rept-sci-L2_20130318_v3.1.0. cdf, rbspa_rel02_ect-rept-sci-L2 20130319_v3.1.0.cdf,rbspb_rel02_ ect-rept-sci-L2_20130316_v3.1.0. cdf, rbspb_rel02_ect-rept-sci-L2_ 20130317_v3.1.0.cdf,rbspb_rel02_ ect-rept-sci-L2_20130318_v3.1.0. cdf, rbspb_rel02_ect-rept-sci-L2_ 20130319_v3.1.0.cdf,rbspa_rel02_ ect-mageis-L2_20130317_v3.0.0.cdf, and rbspa_rel02_ect-mageis-L2 20130318_v3.0.0.cdf; EMFSIS, http://emfisis.physics.uiowa.edu/ Flight/,rbspa_WFR-spectral-matrix_ emfisis-L2_20130317_v1.2.4.cdf, and rbspa_WFR-spectral-matrix emfisis-L2_20130318_v1.3.3.cdf. This work is supported by 973 Program 2012CB825603, the National Natural Science Foundation of China grants 41274165, 40925014, and 41204114, the Aid Program for Science and Technology Innovative Research Team in Higher Educational Institutions of Hunan Province, and the Construct Program of the Key Discipline in Hunan Province. This work was also supported from JHU/APL contract No. 921647 and 967399 under NASA Prime contract No. NAS5-01072.

Michael Balikhin thanks the reviewers for their assistance in evaluating this paper.

\section{References}

Albert, J. M. (2004), Using quasi-linear diffusion to model acceleration and loss from wave-particle interactions, Space Weather, 2(9), S09S03, doi:10.1029/2004SW000069.

Albert, J. M., and S. L. Young (2005), Multidimensional quasi-linear diffusion of radiation belt electrons, Geophys. Res. Lett., 32, L14110, doi:10.1029/2005GL023191.

Baker, D. N., et al. (2012), The relativistic electron-proton telescope (REPT) instrument on board the Radiation Belt Storm Probes (RBSP) spacecraft: Characterization of Earth's radiation belt high-energy particle populations, Space Sci. Rev., 179, 337-381, doi:10.1007/s11214-012-9950-9.

Baker, D. N., et al. (2013), A long-lived relativistic electron storage ring embedded in Earth's outer Van Allen belt, Science, 340, 186-190, doi:10.1126/science.1233518

Brautigam, D. H., and J. M. Albert (2000), Radial diffusion analysis of outer radiation belt electrons during the October 9,1990, magnetic storm, J. Geophys. Res., 105, 291-309.

Ding, Y., Z. He, Z. Zhang, and F. Xiao (2013), Influence of wave normal angle on gyroresonance between chorus waves and outer radiation belt electrons, Sci. China Tech. Sci., 56, 2681-2689, doi:10.1007/s11431-013-5363-6.

Glauert, S. A., and R. B. Horne (2005), Calculation of pitch angle and energy diffusion coefficients with the PADIE code, J. Geophys. Res., 110, A04206, doi:10.1029/2004JA010851.

Horne, R. B., S. A. Glauert, and R. M. Thorne (2003), Resonant diffusion of radiation belt electrons by whistler mode chorus, Geophys. Res. Lett., 30(9), 1493, doi:10.1029/2003GL016963.

Horne, R. B., R. M. Thorne, S. A. Glauert, J. M. Albert, N. P. Meredith, and R. R. Anderson (2005a), Timescale for radiation belt electron acceleration by whistler mode chorus waves, J. Geophys. Res., 110, A03225, doi:10.1029/2004JA010811.

Horne, R. B., et al. (2005b), Wave acceleration of electrons in the Van Allen radiation belts, Nature, 437, 227, doi:10.1038/nature03939.

Kataoka, R., and Y. Miyoshi (2008), Magnetosphere inflation during the recovery phase of geomagnetic storms as an excellent magnetic confinement of killer electrons, Geophys. Res. Lett., 35, L06S09, doi:10.1029/2007GL031842.

Kletzing, C. A., et al. (2013), The Electric and Magnetic Field Instrument Suite and Integrated Science (EMFISIS) on RBSP, Space Sci. Rev, 179, 127-181, doi:10.1007/s11214-013-9993-6.

Kozyra, J. U., C. E. Rasmussen, R. H. Miller, and L. R. Lyons (1994), Interaction of ring current and radiation belt protons with ducted plasmaspheric hiss: 1. Diffusion coefficients and timescales, J. Geophys. Res., 99, 4069-4084.

$\mathrm{Li}$, W., et al. (2013), An unusual enhancement of low-frequency plasmaspheric hiss in the outer plasmasphere associated with substorm-injected electrons, Geophys. Res. Lett., 40, 3798-3803, doi:10.1002/grl.50787.

$\mathrm{Li}$, X., et al. (1997), Multisatellite observations of the outer zone electron variation during the November 3-4, 1993, magnetic storm, J. Geophys. Res., 102(14), 14,123-14,140.

Li, X., A. B. Barker, D. N. Baker, W. C. Tu, T. E. Sarris, R. S. Selesnick, R. Friedel, and C. Shen (2009), Modeling the deep penetration of outer belt electrons during the "Halloween" magnetic storm in 2003, Space Weather, 7, S02004, doi:10.1029/2008SW000418.

Lin, R. L., X. X. Zhang, S. Q. Liu, Y. L. Wang, and J. C. Gong (2010), A three-dimensional asymmetric magnetopause model, J. Geophys. Res. 115, A04207, doi:10.1029/2009JA014235.

Lyons, L. R., R. M. Thorne, and C. F. Kennel (1972), Pitch-angle diffusion of radiation belt electrons within the plasmasphere, J. Geophys. Res., 77, 3455-3474.

Mauk, B. H., N. J. Fox, S. G. Kanekal, R. L. Kessel, D. G. Sibeck, and A. Ukhorskiy (2012), Science objectives and rationale for the radiation belt storm probes mission, Space Sci. Rev., 179, 3-27, doi:10.1007/s11214-012-9908-y.

Morley, S. K., M. G. Henderson, G. D. Reeves, R. H. W. Friedel, and D. N. Baker (2013), Phase space density matching of relativistic electrons using the Van Allen Probes: REPT results, Geophys. Res. Lett., 40, 4798-4802, doi:10.1002/grl.50909.

Reeves, G. D., et al. (2013), Electron acceleration in the heart of the Van Allen radiation belts, Science, 341, 991-994, doi:10.1126/science.1237743

Sheeley, B. W., M. B. Moldwin, H. K. Rassoul, and R. R. Anderson (2001), An empirical plasmasphere and trough density model: CRRES observations, J. Geophys. Res., 106, 25,631-25,641.

Shprits, Y. Y., D. Subbotin, A. Drozdov, M. E. Usanova, A. Kellerman, K. Orlova, D. N. Baker, D. L. Turner, and K.-C. Kim (2013), Unusual stable trapping of the ultrarelativistic electrons in the Van Allen radiation belts, Nat. Phys., 9, 699-703, doi:10.1038/nphys2760.

Summers, D., R. M. Thorne, and F. Xiao (1998), Relativistic theory of wave-particle resonant diffusion with application to electron acceleration in the magnetosphere, J. Geophys. Res., 103, 20,487-20,500.

Tao, X., A. A. Chan, J. M. Albert, and J. A. Miller (2008), Stochastic modeling of multi-dimensional diffusion in the radiation belts, J. Geophys. Res., 113, A07212, doi:10.1029/2007JA012985.

Thorne, R. M. (2010), Radiation belt dynamics: The importance of wave-particle interactions, Geophys. Res. Lett., 37, L22107, doi:10.1029/2010GL044990.

Thorne, R. M., et al. (2013a), Evolution and slow decay of an unusual narrow ring of relativistic electrons near $L \sim 3.2$ following the September 2012 magnetic storm, Geophys. Res. Lett., 40, 3507-3511, doi:10.1002/grl.50627.

Thorne, R. M., et al. (2013b), Rapid local acceleration of relativistic radiation-belt electrons by magnetospheric chorus, Nature, 504, 411-414, doi:10.1038/nature12889.

Tu, W. C., X. Li, Y. Chen, G. D. Reeves, and M. Temerin (2009), Storm-dependent radiation belt electron dynamics, J. Geophys. Res., 114, A02217, doi:10.1029/2008JA013480.

Vasyliunas, V. M. (1968), A survey of low-energy electrons in the evening sector of the magnetosphere with OGO 1 and OGO 3, J. Geophys. Res., 73, 2839-2884.

Viñas, A. F., R. L. Mace, and R. F. Benson (2005), Dispersion characteristics for plasma resonances of Maxwellian and kappa distribution plasmas and their comparisons to the image/rpi observations, J. Geophys. Res., 110, A06202, doi:10.1029/2004JA010967.

Wygant, J. R., et al. (2013), The Electric Field and Waves (EFW) instruments on the Radiation Belt Storm Probes Mission, Space Sci. Rev., 179, 183-220, doi:10.1007/s11214-013-0013-7.

Xiao, F. (2006), Modelling energetic particles by a relativistic kappa loss cone distribution function in plasmas, Plasma Phys. Controlled Fusion, 48, 203.

Xiao, F., C. Shen, Y. Wang, H. Zheng, and S. Wang (2008), Energetic electron distributions fitted with a relativistic kappa-type function at geosynchronous orbit, J. Geophys. Res., 113, A05203, doi:10.1029/2007JA012903.

Xiao, F., Z. Su, H. Zheng, and S. Wang (2009), Modeling of outer radiation belt electrons by multidimensional diffusion process, J. Geophys. Res., 114, A03201, doi:10.1029/2008JA013580.

Xiao, F., Z. Su, H. Zheng, and S. Wang (2010), Three-dimensional simulations of outer radiation belt electron dynamics including cross diffusion terms, J. Geophys. Res., 115, A05216, doi:10.1029/2009JA014541. 